\title{
Bactericidal efficacy of meropenem in combination with cefmetazole against IMP-producing carbapenem-resistant Enterobacteriaceae
}

Ryuichiro Abe ${ }^{1,2,3}$, Hideharu Hagiya ${ }^{1,4^{*}}$ (D), Yukihiro Akeda ${ }^{1,2}$, Norihisa Yamamoto ${ }^{1}$, Yoshikazu Ishii ${ }^{5}$ and Kazunori Tomono ${ }^{1}$

\begin{abstract}
Objective: Carbapenem-resistant Enterobacteriaceae (CRE) are among the most severe threats to public and clinical health because of their high levels of resistance to various antibiotics. We assessed the efficacy of combination therapy with meropenem (MEM) and cefmetazole (CMZ) against Imipenemase (IMP)-producing CRE, using the checkerboard method and time-killing assay on 13 Enterobacteriaceae isolates harboring bla IMP-1 $_{1}$ (4 Enterobacter hormaechei, 5 Escherichia coli, and 4 Klebsiella pneumoniae isolates) and 13 isolates harboring bla $a_{\mathrm{IMP}-6}$ (8 E. coli and 5 K. pneumoniae isolates).

Results: Minimum inhibitory concentrations (MICs) of MEM and CMZ ranged from 2 to 64 and 64 to $2048 \mu \mathrm{g} / \mathrm{mL}$, respectively. Checkerboard method demonstrated the synergy of the MEM/CMZ combination in all the tested IMPproducing CRE isolates, and the time-kill assay indicated a bactericidal effect for both bla $a_{\mid \mathrm{MP}-1}$ and bla $a_{\mathrm{IMP}-6}$ positive CRE when MEM/CMZ combination was used. In vitro, the MEM/CMZ combination was potentially effective against IMP-1or IMP-6-producing CRE. Further investigations including in vivo animal studies and clinical studies are warranted to corroborate the clinical utility of the novel combination therapy.
\end{abstract}

Keywords: Carbapenem-resistant Enterobacteriaceae, Carbapenemase-producing Enterobacteriacea, Cephamycin, Cefmetazole, IMP

\section{Introduction}

Carbapenems are the last-resort antibiotics for the treatment of various infections caused by multidrug-resistant gram-negative bacteria. The wide global dissemination of carbapenem-resistant Enterobacteriaceae (CRE) is a serious threat to global public health and is a major concern to clinicians. Carbapenem resistance is mainly due to the production of carbapenemases whose genes are encoded on plasmids that are transmitted easily across bacterial

\footnotetext{
*Correspondence: hagiya@okayama-u.ac.jp

${ }^{1}$ Department of Infection Control and Prevention, Osaka University Graduate School of Medicine, 2-15 Yamadaoka, Suita, Osaka 565-0871, Japan

Full list of author information is available at the end of the article
}

species, which has resulted in the rapid spread of CRE worldwide [1].

Carbapenemases are categorized into mainly three classes (classes A, B, and D) of the Amber $\beta$ lactamase classification. The class A Klebsiella pneumoniae carbapenemases (KPC) are one of the most prevalent carbapenamases. The class D Oxacillin-hydrolysing (OXA)-48 carbapenemase producers have disseminated globally. Class B metallo- $\beta$-lactamases (MBLs) mainly comprise the Verona Integron-Mediated (VIM)-, Imipenemase (IMP)-, and New Delhi Metallo-beta-lactamase (NDM)types. Of these, NDM producers have rapidly spread worldwide since the first reports of their emergence in 2009 [1]. Despite their global dissemination, KPCs, OXAs, and NDMs are still not common in Japan, where 
IMP-1 and IMP-6 are the exclusively predominant carbapenemases [2, 3].

Treatment options for patients infected with carbapenemase-producing Enterobacteriaceae (CPE) are limited. For class A carbapenemases, such as KPC, a double-carbapenem therapy has been used [4]. Additionally, ceftazidime/avibactam combination, which is officially approved in Europe and the United States but not in Japan, has exhibited potent activity against OXA- 48 producing gram-negative organisms [5]. However, MBL producers are resistant to these new treatments, and few studies have explored treatment strategies for MBL-producing Enterobacteriaceae [5, 6].

In a previous study, we demonstrated the in vitro efficacy of the combination of meropenem (MEM) and cefmetazole (CMZ) against KPC producers [7]. In this study, we investigated the in vitro activity of the MEM/ CMZ combination therapy against IMP-producing CRE.

\section{Main text Methods}

We used 13 Enterobacteriaceae isolates harboring

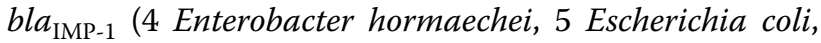
and 4 Klebsiella pneumoniae) provided by Toho University, and 13 Enterobacteriaceae isolates harboring bla $_{\text {IMP-6 }}$ (8 E. coli and $5 \mathrm{~K}$. pneumoniae) obtained in our previous CRE surveillance in Osaka, Japan [2]. E. hormaechei isolates belonging to Enterobacter cloacae complex were included in this analysis as one of $b l a_{\mathrm{IMP}-1}$ representative carriers, which were detected in an outbreak reported in Tokyo, Japan [3]. Through the experiments explained below, we used meropenem trihydrate (Tokyo Chemical Industry, Japan, Tokyo) and cefmetazole sodium salt (Sigma-Aldrich, Saint Louis, MO, United States) as antibiotic agents.

We determined minimum inhibitory concentrations (MICs) of MEM and CMZ by the broth microdilution method based on the Clinical and Laboratory Standards Institute (CLSI) document M07-A10 [8]. Bacterial growth was evaluated by visible observation for turbidity. Briefly, we inoculated $5 \times 10^{5}$ colony forming units $(\mathrm{CFU}) / \mathrm{mL}$ of bacterial suspension into cation-adjusted $\mathrm{BBL}^{\mathrm{TM}}$ Mueller-Hinton II Broth (Becton, Dickinson and Company, Sparks, MD, USA) and incubated at $35^{\circ} \mathrm{C}$ in ambient air for $18 \mathrm{~h}$. MIC was defined as the lowest concentration of the tested antimicrobial that completely inhibited the growth of bacteria.

Details of the checkerboard method and time-killing assay have been presented previously [7]. In the checkerboard method, synergistic effect between MEM and CMZ against IMP-1 or IMP-6 producers was quantified by calculating the fractional inhibitory concentration (FIC) index. FIC index value $\leq 0.5$ was defined as synergy,
$>0.5$ to $\leq 4.0$ as indifferent, and $>4.0$ as antagonistic. The assay was performed in duplicate. In case a synergistic effect was observed, MEM MIC fold-reduction was determined based on the lowest FIC index.

We conducted time-killing assays using the following two E. coli isolates: TUM13773 carrying bla $_{\mathrm{IMP}-1}$ and E109 carrying $b$ I IMP-6 $_{\text {. }}$. During these assays, each isolate was incubated in Muller-Hinton II broth devoid of antibiotic (control) and with MEM, CMZ, or MEM/CMZ combination at the concentration of $25 \%$ of the MIC for individual isolates. CFUs of bacteria at 3, 6, 9, and $24 \mathrm{~h}$ after beginning the treatment were counted. The averages of CFUs were calculated from duplicated assays. We defined an efficacy of the combination therapy as bactericidal when $\geq 3 \log _{10} \mathrm{CFU} / \mathrm{mL}$ reduction compared to the initial bacterial count was observed.

\section{Results}

MIC ranges of MEM and CMZ for isolates harboring $b l a_{\mathrm{IMP}-1}$ were 2 to $8 \mu \mathrm{g} / \mathrm{mL}$ and 64 to $2048 \mu \mathrm{g} / \mathrm{mL}$, respectively. Similarly, those harboring $b l a_{\mathrm{IMP}-6}$ ranged from 16 to $32 \mu \mathrm{g} / \mathrm{mL}$ and from 64 to $1024 \mu \mathrm{g} / \mathrm{mL}$, respectively (Table 1). No isolates were susceptible to both MEM and CMZ based on the CLSI breakpoint values [9].

The checkerboard method showed that the combination of MEM and CMZ was synergistically active against all the tested IMP-producing CRE isolates (Table 1). MEM MICs in the combination achieved 4- to 32-fold reduction of the MIC of MEM alone.

In the time-killing assays, the viable bacteria treated with each antibiotic alone regrew at $24 \mathrm{~h}$ (Fig. 1). Contrarily, upon using the combination of $0.25 \times$ MIC MEM and $0.25 \times$ MIC CMZ, no growth was detected at $9 \mathrm{~h}$ and $24 \mathrm{~h}$, indicating a bactericidal effect against the tested isolates.

\section{Discussion}

We herein demonstrated the in vitro efficacy of MEM in combination with CMZ against IMP-producing CRE isolates. In addition to the double-carbapenem therapy [4] and ceftazidime/avibactam [5], newer $\beta$-lactamase inhibitors including relebactam (MK-7655), nacubactam (OP0595), zidebactam (WCK 5107), and vaborbactam (RPX7009) have been developed to treat CPE that produce serine-carbapenemases [10]. On the other hand, there is less available treatment option for infections caused by MBL-producing CRE.

Still much remains to be done, this investigation is very novel to the literature, in that it was intended to corroborate the potency of CMZ to cancel the function of IMP-type carbapenemases. CMZ is one of the established antimicrobials with accumulated data of its use. It is a cephamycin antibiotic that is stable against 
Table 1 Minimum inhibitory concentrations (MICs) and fractional inhibitory concentration (FIC) index of b/a $a_{\text {IMP-1 }}$ or bla ${ }_{\text {IMP- } 6}$ positive Enterobacteriaceae isolates

\begin{tabular}{|c|c|c|c|c|c|c|c|}
\hline & \multirow[t]{2}{*}{ Isolates } & & \multicolumn{2}{|c|}{ MIC $(\mu \mathrm{g} / \mathrm{mL})$} & \multirow[t]{2}{*}{ MEM MIC reduction } & \multirow[t]{2}{*}{ FIC index } & \multirow[t]{2}{*}{ Evaluation } \\
\hline & & & MEM & $\mathrm{CMZ}$ & & & \\
\hline \multirow[t]{13}{*}{ IMP-1 producing isolates } & TUM10695 & E. hormaechei & 2 & 512 & $1 / 4,1 / 8$ & $0.27,0.19$ & Synergy \\
\hline & TUM11051 & E. hormaechei & 8 & 2048 & $1 / 4,1 / 4$ & $0.50,0.50$ & Synergy \\
\hline & TUM11052 & E. hormaechei & 8 & 1024 & $1 / 4,1 / 4$ & $0.28,0.27$ & Synergy \\
\hline & TUM11134 & E. hormaechei & 2 & 1024 & $1 / 4,1 / 4$ & $0.31,0.50$ & Synergy \\
\hline & TUM11259 & E. coli & 8 & 512 & $1 / 8,1 / 8$ & $0.25,0.38$ & Synergy \\
\hline & TUM13773 & E. coli & 4 & 256 & $1 / 4,1 / 8$ & $0.50,0.37$ & Synergy \\
\hline & TUM14683 & E. coli & 4 & 512 & $1 / 16,1 / 8$ & $0.19,0.19$ & Synergy \\
\hline & TUM14697 & E. coli & 2 & 64 & $1 / 4,1 / 8$ & $0.38,0.19$ & Synergy \\
\hline & TUM14759 & E. coli & 4 & 512 & $1 / 8,1 / 4$ & $0.25,0.28$ & Synergy \\
\hline & TUM13774 & K.pneumoniae & 8 & 256 & $1 / 8,1 / 8$ & $0.25,0.38$ & Synergy \\
\hline & TUM13775 & K.pneumoniae & 8 & 512 & $1 / 8,1 / 8$ & $0.25,0.25$ & Synergy \\
\hline & TUM14366 & K.pneumoniae & 8 & 256 & $1 / 8,1 / 4$ & $0.25,0.31$ & Synergy \\
\hline & TUM14380 & K.pneumoniae & 2 & 512 & $1 / 4,1 / 4$ & $0.50,0.38$ & Synergy \\
\hline \multirow[t]{13}{*}{ IMP-6 producing isolates } & E015 & E. coli & 16 & 256 & $1 / 8,1 / 16$ & $0.16,0.13$ & Synergy \\
\hline & E020 & E. coli & 16 & 256 & $1 / 8,1 / 16$ & $0.13,0.19$ & Synergy \\
\hline & E030 & E. coli & 32 & 512 & $1 / 16,1 / 16$ & $0.09,0.08$ & Synergy \\
\hline & E038 & E. coli & 16 & 512 & $1 / 32,1 / 16$ & $0.05,0.14$ & Synergy \\
\hline & E046 & E. coli & 32 & 1024 & $1 / 8,1 / 8$ & $0.14,0.14$ & Synergy \\
\hline & E070 & E. coli & 16 & 256 & $1 / 32,1 / 32$ & $0.19,0.06$ & Synergy \\
\hline & E109 & E. coli & 16 & 64 & $1 / 32,1 / 32$ & $0.28,0.28$ & Synergy \\
\hline & E138 & E. coli & 64 & 1024 & $1 / 8,1 / 8$ & $0.19,0.19$ & Synergy \\
\hline & E039 & K.pneumoniae & 32 & 256 & $1 / 16,1 / 16$ & $0.13,0.13$ & Synergy \\
\hline & E045 & K.pneumoniae & 32 & 256 & $1 / 8,1 / 16$ & $0.16,0.09$ & Synergy \\
\hline & E065 & K.pneumoniae & 32 & 256 & $1 / 32,1 / 32$ & $0.09,0.06$ & Synergy \\
\hline & E085 & K.pneumoniae & 32 & 512 & $1 / 8,1 / 8$ & $0.19,0.16$ & Synergy \\
\hline & E139 & K. pneumoniae & 32 & 1024 & $1 / 8,1 / 4$ & $0.25,0.27$ & Synergy \\
\hline
\end{tabular}

CMZ cefmetazole, MEM meropenem

FIC index was measured in duplication. FIC index was calculated in following formula; FIC index $=$ (MIC of MEM measured in combination with CMZ)/(MIC of MEM only) + (MIC of CMZ measured in combination with MEM)/(MIC of CMZ only). FIC index $\leq 0.5$ was defined as synergy, $>0.5$ to $\leq 4.0$ as indifferent, and $>4.0$ as antagonistic. MEM MIC fold-reduction by CMZ was calculated at the lowest FIC index

a IMP-1 producing E. coli

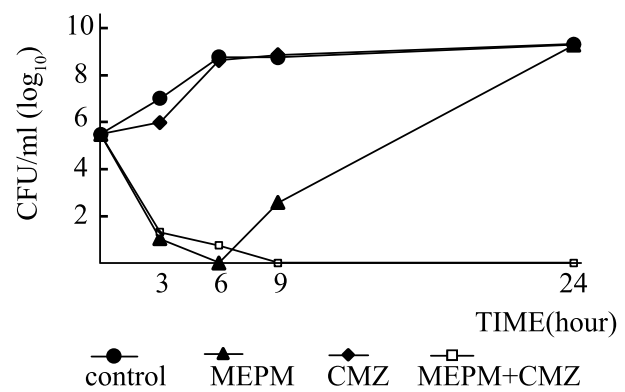

b IMP-6 producing E. coli

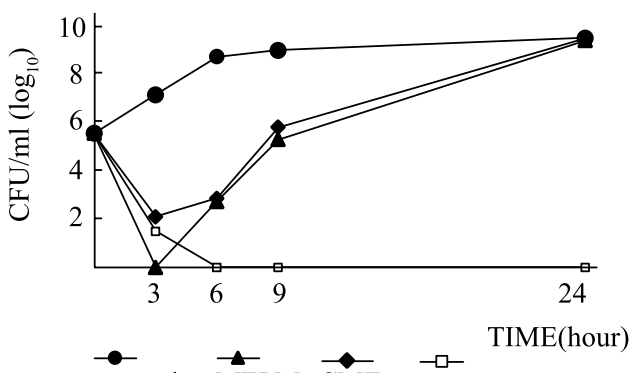

$\stackrel{\bullet}{\text { control }} \overrightarrow{\mathrm{MEPM}} \quad \overrightarrow{\mathrm{CMZ}} \quad \stackrel{-}{\mathrm{MEPM}}+\mathrm{CMZ}$

Fig. 1 Time kill curves for IMP-1- or IMP-6-producing E. coli isolates with MEM or CMZ only, or both MEM and CMZ. E. coli isolate TUM13773 carrying bla $a_{1 \mathrm{MP}-1.1}$ a and E. coli isolate E109 carrying bla $a_{\mathrm{IMP}-6}$. b were incubated in Mueller-Hinton II Broth (black circle) or supplemented with MEM (black up-pointing triangle), with CMZ (black diamond) or with combination of MEM and CMZ (square) at $37{ }^{\circ} \mathrm{C}$. All the antibiotics were used at concentrations of $25 \%$ of their MIC for individual isolates. Viable cells per milliliter before incubation and after incubation for 3, 6, 9, and $24 \mathrm{~h}$ were counted on Muller Hinton II agar plates after overnight incubation at $37^{\circ} \mathrm{C}$. Assays were performed in duplicate and the logarithm of the average $\mathrm{CFU} / \mathrm{mL}$ was plotted 
extended-spectrum $\beta$-lactamases (ESBLs) [11], but is hydrolyzed by carbapenemases, including IMPs [12]. Presently, CRE isolates that produced IMPs were highly resistant to CMZ, with MICs ranging 64 to $2048 \mu \mathrm{g} /$ $\mathrm{mL}$. These findings may indicate a high affinity of CMZ to IMP-type carbapenemases, and is consistent with our hypothesis that CMZ binds avidly to the carbapenemases, helping MEM exert its bactericidal activity, as seen in double-carbapenem therapy [4]. To demonstrate the superiority of CMZ to MEM in terms of affinity to IMP-type carbapenemases, we need to perform a kinetic assay in future study. ESBL producing Enterobacteriaceae have globally disseminated [12] and CRE isolates co-harboring genes encoding ESBLs and carbapenemases have been previously described $[13,14]$. Particularly, $b l a_{\text {IMP-6 }}$ was reported to disseminate mainly through the horizontal transmission of the prevalent plasmid, pKPI-6, which simultaneously carries $b l a_{\mathrm{IMP}-6}$ and $b l a_{\mathrm{CTX}-\mathrm{M} 2}$ [15]. The MEM and CMZ combination therapy would be a readyto-apply, cost-effective strategy for IMP-producing CRE even against producing ESBLs, compared to the newer $\beta$-lactamase inhibitors.

\section{Limitations}

However, several limitations remain to be overcome prior to clinical use. First, the CMZ concentrations tested in this study exceed the serum levels attainable in humans. Second, as shown in our previous study [7], an inoculum effect may exist in this combination therapy, possibly resulting in treatment failure using a high bacterial inoculum. Third, various antimicrobial resistance mechanisms, such as AmpC-type beta-lactamases, efflux pumps, and porin loss, may influence the inhibitory activity of CMZ.

Despite these limitations, the collective data from this study demonstrate a preferential effect of MEM and CMZ when used in combination against IMP-producing CRE in vitro. Faced with the limited availability of new antimicrobials, the revived use of an existing antimicrobial agent could provide effective treatment. In vivo experiments as well as pharmacokinetic and pharmacodynamic studies are required before the clinical application of the new combination therapy.

\footnotetext{
Abbreviations

CFU: colony forming units; CLSI: Clinical and Laboratory Standards Institute; CMZ: cefmetazole; CPE: carbapenemase-producing Enterobacteriaceae; CRE: carbapenem-resistant Enterobacteriaceae; ESBLs: extended-spectrum $\beta$-lactamases; FIC: fractional inhibitory concentration; IMP: imipenemase; KPC: Klebsiella pneumoniae carbapenemases; MBL: metallo- $\beta$-lactamases; MEM: meropenem; MICs: minimum inhibitory concentrations; NDM: New Delhi Metallo-beta-lactamase; OXA: oxacillin-hydrolysing; VIM: Verona Integron-Mediated.
}

Acknowledgements

None.
Authors' contributions

Data acquisition and writing: $\mathrm{RA}$ and $\mathrm{HH}$. Critical revision: $Y A, N Y, Y I$, and $\mathrm{KT}$. All authors read and approved the final manuscript.

\section{Funding}

This work was supported by Grants-in-Aid for Scientific Research (No.

$15 \mathrm{~K} 19585,17 \mathrm{~K} 16223)$ in performing the in vitro experiments.

\section{Availability of data and materials}

Not applicable.

Ethics approval and consent to participate

Not applicable.

Consent to publish

Not applicable.

\section{Competing interests}

The authors declare that they have no competing interests.

\section{Author details}

${ }^{1}$ Department of Infection Control and Prevention, Osaka University Graduate School of Medicine, 2-15 Yamadaoka, Suita, Osaka 565-0871, Japan. ${ }^{2}$ Research Institute for Microbial Diseases, Osaka University, Osaka, Japan. ${ }^{3}$ Department of Anesthesiology and Intensive Care Medicine, Graduate School of Medicine, Osaka University, Osaka, Japan. ${ }^{4}$ Department of General Medicine, Graduate School of Medicine Dentistry, and Pharmaceutical Sciences, Okayama University, Okayama, Japan. ${ }^{5}$ Department of Microbiology and Infectious Diseases, Toho University School of Medicine, Tokyo, Japan.

Received: 30 September 2019 Accepted: 1 November 2019

Published online: 09 November 2019

\section{References}

1. Logan LK, Weinstein RA. The epidemiology of carbapenem-resistant enterobacteriaceae: the impact and evolution of a global menace. $J$ Infect Dis. 2017;215:S28-36.

2. Yamamoto N, Asada R, Kawahara R, Hagiya H, Akeda Y, Shanmugakani RK, et al. Prevalence of, and risk factors for, carriage of carbapenem-resistant Enterobacteriaceae among hospitalized patients in Japan. J Hosp Infect. 2017;97:212-7

3. Aoki K, Harada S, Yahara K, Ishii Y, Motooka D, Nakamura S, et al. Molecular characterization of IMP-1-producing enterobacter cloacae complex isolates in Tokyo. Antimicrob Agents Chemother. 2018;62:e0209117.

4. Giamarellou H, Galani L, Baziaka F, Karaiskos I. Effectiveness of a doublecarbapenem regimen for infections in humans due to carbapenemaseproducing pandrug-resistant Klebsiella pneumoniae. Antimicrob Agents Chemother. 2013;57:2388-90.

5. Kazmierczak KM, de Jonge BLM, Stone GG, Sahm DF. In vitro activity of ceftazidime/avibactam against isolates of Enterobacteriaceae collected in European countries: INFORM global surveillance 2012-15. J Antimicrob Chemother. 2018;73:2782-8.

6. Tangden T, Hickman RA, Forsberg P, Lagerback P, Giske CG, Cars O. Evaluation of double- and triple-antibiotic combinations for VIM- and NDM-producing Klebsiella pneumoniae by in vitro time-kill experiments. Antimicrob Agents Chemother. 2014;58:1757-62.

7. Hagiya H, Aoki K, Akeda Y, Yamamoto N, Shanmugakani RK, Ishii Y, et al. In vitro effectiveness of meropenem and cefmetazole combination treatment against KPC-2-producing Enterobacteriaceae. Microb Drug Resist. 2019;25:839-45.

8. Clinical and Laboratory Standards Institute (CLSI). M07-A10. Methods for dilution antimicrobial susceptibility tests for bacteria that grow aerobically: approved standards. 10th ed. Wayne: CLSI; 2015.

9. Clinical and Laboratory Standards Institute. M100-S28. Performance standards for antimicrobial susceptibility testing. Wayne: CLSI; 2018.

10. Bowers DR, Huang V. Emerging issues and treatment strategies in carbapenem-resistant Enterobacteriaceae (CRE). Curr Infect Dis Rep. 2016;18:48. 
11. Doi A, Shimada T, Harada S, Iwata K, Kamiya T. The efficacy of cefmetazole against pyelonephritis caused by extended-spectrum beta-lactamaseproducing Enterobacteriaceae. Int J Infect Dis. 2013;17:e159-63.

12. Jacoby GA, Munoz-Price LS. The new beta-lactamases. N Eng J Med. 2005;352:380-91.

13. Huang W, Wang G, Sebra R, Zhuge J, Yin C, Aguero-Rosenfeld ME, et al. Emergence and evolution of multidrug-resistant Klebsiella pneumoniae with both bla $a_{\mathrm{KPC}}$ and bla $a_{\mathrm{CTX}-\mathrm{M}}$ integrated in the chromosome. Antimicrob Agents Chemother. 2017:61:e00076-17.

14. Rojas LJ, Weinstock GM, De La Cadena E, Diaz L, Rios R, Hanson BM, Brown $\mathrm{JS}$, et al. An analysis of the epidemic of Klebsiella pneumoniae carbapenemase-producing $K$. pneumoniae: convergence of two evolutionary mechanisms creates the "Perfect Storm". J Infect Dis. 2017;217:82-92.
15. Kayama S, Shigemoto N, Kuwahara R, Oshima K, Hirakawa H, Hisatsune J, et al. Complete nucleotide sequence of the IncN plasmid encoding IMP- 6 and CTX-M-2 from emerging carbapenem-resistant Enterobacteriaceae in Japan. Antimicrob Agents Chemother. 2015;59:1356-9.

\section{Publisher's Note}

Springer Nature remains neutral with regard to jurisdictional claims in published maps and institutional affiliations.
Ready to submit your research? Choose BMC and benefit from:

- fast, convenient online submission

- thorough peer review by experienced researchers in your field

- rapid publication on acceptance

- support for research data, including large and complex data types

- gold Open Access which fosters wider collaboration and increased citations

- maximum visibility for your research: over $100 \mathrm{M}$ website views per year

At BMC, research is always in progress.

Learn more biomedcentral.com/submissions 